\title{
Desempenho de indicadores nos municípios com alta cobertura da Estratégia Saúde da Família no Estado de São Paulo
}

\author{
Performance of Indicators in municipalities with High Coverage of the Family \\ Health Strategy in the State of São Paulo
}

Desempeño de indicadores en los municipios con una alta cobertura de la "Estrategia de Salud Familiar" en el Estado de São Paulo

Rodrigo de Novaes Lima ${ }^{1 *}$, Martim Elviro de Medeiros Junior², Julie Silvia Martins³,

Eduardo Pires dos Santos ${ }^{4}$, Monique Marie Marthe Bourget ${ }^{5}$

Palavras-chave: Avaliação de Serviços de Saúde Indicadores Básicos de Saúde Indicadores de Morbimortalidade Atenção Primária à Saúde Estratégia Saúde da Família

\section{Resumo}

Introdução: A avaliação do desempenho da Estratégia Saúde da Família tem se mostrado difícil no Estado de São Paulo por razões diversas, como a baixa cobertura da população, associada paradoxalmente a indicadores de saúde acima da média nacional. Objetivo: Avaliar 0 desempenho de alguns indicadores do "Pacto pela Atenção Básica" e do "Pacto pela Saúde" em municípios do Estado de São Paulo com alta cobertura da Estratégia Saúde da Família em comparação ao restante do Estado. Métodos: Indicadores do "Pacto pela Atenção Básica" e do "Pacto pela Saúde" foram acompanhados nos 110 municípios que alcançaram 90\% ou mais de cobertura da Estratégia Saúde da Família em um período mínimo de sete anos e comparados com os indicadores do restante do Estado. Resultados: A taxa de mortalidade infantil apresentou uma tendência de queda muito mais importante em relação ao Estado. A taxa de internação por acidente vascular cerebral mostrou-se maior nos municípios em relação ao Estado. A razão de exames citopatológicos dos municípios da amostra foi quase 0 dobro da encontrada no Estado; a taxa de alta dos pacientes com tuberculose foi superior em pouco mais de 13\% em comparação à média do Estado e a taxa de cura de hansênicos apresentou variação menor (5\%), mas favorável à amostra. Conclusões: 0s achados sugerem que a estratégia esteja contribuindo para a melhora dos indicadores de processo, bem como de alguns indicadores de resultado, demandando maior seguimento dos últimos.

\footnotetext{
Casa de Saúde Santa Marcelina. rodrigonovaeslma@yahoo.com.br

${ }^{2}$ Casa de Saúde Santa Marcelina. elvirojr@ig.com.br

${ }^{3}$ Casa de Saúde Santa Marcelina. julie@aps.santamarcelina.org

${ }^{4}$ Casa de Saúde Santa Marcelina. duzinho_pires@yahoo.com.br

${ }^{5}$ Casa de Saúde Santa Marcelina. monique@santamarcelina.org

*Autor correspondente.

Fonte de financiamento: Casa de Saúde Santa Marcelina.

Conflito de interesses: declaram não haver.

Recebido em: 17/06/2011

Aprovado em: 18/10/2012
} 


\begin{abstract}
Keywords: Abstract
Health Services Evaluation

Health Status Indicators

Indicators of Morbidity and Mortality

Primary Health Care

Family Health Strategy

Objective: It has been difficult to evaluate the performance of the Family Health Strategy in the State of São Paulo due to several reasons, such as the low population covered, which is paradoxically associated to health indicators above the national average. On this basis, this study tries to evaluate the strategy effectiveness in the State of São Paulo. Methods: The indicators of the Primary Care Pact and Health Pact were investigated in the municipalities that achieved $90 \%$ or more coverage of the Family Health Strategy for the past seven years. Results: Infant mortality presented a more important downward trend within the sample. The hospitalization rate for stroke was higher within the sample, but presented stability that opposes the growth in the state. The cytopathologic exams ratio was nearly double the amount found elsewhere in the State. The cure rate for tuberculosis patients was $13 \%$ higher than the state pattern and the cure rate of leprosy showed minor variation (5\%), but favorable to the cities of the sample. Conclusions: The findings have led to the conclusion that the strategy demonstrated improvement in the process indicators, as well as in the results of some of the indicators studied, demanding a longer follow-up for better conclusions.
\end{abstract}

Palabras clave:

Evaluación de Servicios de Salud Indicadores Básicos de Salud Indicadores de Morbimortalidad Atención Primaria de la Salud Estrategia de Salud Familiar

\section{Resumen}

Introducción: Evaluar el desempeño de la "Estrategia de Salud Familiar" (ESF) en el estado de São Paulo ha sido difícil por diversas razones, como por ejemplo la baja cobertura de la población, asociada, paradójicamente, a indicadores de salud superiores al promedio nacional. Objetivo: Evaluar el desempeño de algunos indicadores del "Pacto por la Atención Básica" y del "Pacto por La Salud" en municipios del Estado de São Paulo con alta cobertura de la ESF con relación a otros municipios del Estado. Métodos: Los indicadores del "Pacto por la Atención Básica" y del "Pacto por La Salud" fueron seguidos en 110 municipios con 90\% o más de cobertura de la ESF, durante un período mínimo de siete años y fueron comparados con los indicadores de los demás municipios del Estado. Resultados: La taxa de mortalidad infantil decreció en la muestra mucho más que en el resto del Estado. La tasa de hospitalización por accidente cerebrovascular fue mayor en la muestra que en el resto del Estado. La proporción de exámenes citopatológicos de los municipio de la muestra fue casi el doble que la encontrada en el resto del Estado; la tasa de alta de los pacientes con tuberculosis fue superior en poco más del 13\% cuando comparada a la media del resto del Estado y la tasa de cura de leprosos mostró una menor variación (5\%), más favorable a la muestra. Conclusiones: Los resultados sugieren que la ESF puede contribuir con la mejora en los indicadores de procesos, así como de algunos de los indicadores de resultados, requiriendo estos últimos mayor seguimiento.

\section{Introdução}

A Estratégia Saúde da Família (iniciada em 1994 como Programa Saúde da Família a partir da experiência exitosa do Programa de Agentes Comunitários de Saúde (PACS) em Quixadá-CE) teve grande expansão em seus 17 anos de história, alcançando, em 2011, uma cobertura nacional de $49,21 \%{ }^{4}$, com regiōes, como a Nordeste, possuindo $71,84 \%$ da população cadastrada na estratégia, ${ }^{4}$ e contando com mais de 245 mil agentes comunitários de saúde 5 . A atenção primária bem desenvolvida tem melhorado os perfis de morbimortalidade em inúmeros países ${ }^{6}$. Da mesma forma, desde o seu surgimento, também se observaram publicações várias no País, demonstrando a efetividade da Estratégia Saúde da Família (ESF) na melhora dos perfis de saúde nacionais ${ }^{5,7-11}$, com perceptível queda de coeficientes de mortalidade infantil, bem como redução no número de internaçóes por causas sensíveis à Atenção Primária à Saúde (APS). Outro aspecto importante revelado pela Pesquisa Nacional de Amostra de Domicílios (2008) é que, de 1998 a 2008, observou-se um aumento importante no acesso aos serviços de saúde, seja para procedimentos mais simples, como para procedimentos mais complexos ${ }^{12}$.

Apesar desses avanços, a Estratégia Saúde da Família tem a proporção de 29,26\% da população acompanhada no Estado de São Paulo e, em sua capital, 31,67\% de cobertura ${ }^{4}$. Devido a posicionamentos políticos na época de seu surgimento, sabe-se que, no Estado, a estratégia teve seu início tardiamente. Também lento foi o seu crescimento em São Paulo, não acompanhando a tendência nacional. Tem-se que a estratégia possui cobertura maior em municípios de pequeno e médio porte, e menor cobertura em capitais. Neste sentido, a cidade de São Paulo, com pouco mais de $31 \%$ da populaçáo coberta, encontra cobertura próxima à encontrada nas outras capitais. Por outro lado, o Estado (29,26\%) está à frente do Distrito Federal $(13,38 \%)$ e Rio de Janeiro $(23,92 \%)$ apenas (dados referentes a 2011) $)^{4}$.

Entretanto, o terceiro Estado com menor proporção de famílias cadastradas na Estratégia Saúde da Família apresenta indicadores acima da média nacional. A taxa de mortalidade infantil do Estado no ano de 2010 (11,89 para 1000 nascidos vivos) estava bem abaixo das encontradas nas demais regióes do País, perdendo apenas para Santa Catarina e Rio Grande do Sul. O percentual de crianças menores de 5 anos com baixo peso no Estado (2,74\%), em 2011, encontrava-se abaixo dos valores para a regiáo Sudeste $(3,36 \%)$ e para o País $(4,45 \%)^{4}$.

Os indicadores paulistas, bem se sabe, são fruto de diversos outros fatores relacionados às condiçóes de vida e políticas públicas, tornando-se difícil quantificar os resultados da Estratégia Saúde da Família no Estado. Outro recente estudo 
em capitais nordestinas, também procurando mensurar o papel da estratégia, não encontrou resultados expressivos, creditando à estratégia grandes mudanças nos perfis de morbimortalidade em cidades menores do País e resultados mais modestos nas capitais, talvez devido às numerosas variáveis macroeconômicas envolvidas no processo saúde-doença dos grandes centros urbanos ${ }^{13}$.

O objetivo do presente trabalho é avaliar o desempenho dos indicadores de saúde propostos no Pacto da Atenção Básica e Pacto pela Saúde 2010/2011 em cidades do Estado de São Paulo que apresentam cobertura igual ou superior a $90 \% \mathrm{da}$ população na "Estratégia Saúde da Família" comparando-as às demais regióes do Estado.

\section{Métodos}

A fim de se mensurar a ação da Estratégia Saúde da Família em São Paulo, foi delineado um estudo ecológico longitudinal baseado na série histórica dos indicadores do Pacto pela Atenção Básica ${ }^{14}$ e do Pacto pela Saúde ${ }^{15}$, política colocada hoje como prioritária para estados e municípios pelo Ministério da Saúde. Do mesmo modo, o trabalho pode ser considerado como um estudo de análise de tendência dos indicadores de ambos os pactos supracitados. Desta forma, foram avaliados dados dos municípios que obtiveram 90\% ou mais de cobertura da Estratégia Saúde da Família por um período mínimo de sete anos (110 ao todo no estado), dados estes comparados com os achados do Estado de Sáo Paulo. O grupo de comparação incluiu todos os municípios do Estado, inclusive aqueles da amostra. Numa tentativa anterior, coberturas inferiores a $90 \%$ foram avaliadas, com desfechos menos expressivos. Da mesma forma, o critério de tempo (7 anos) foi assim determinado porque bem se sabe que alguns indicadores têm resultados mais precoces em decorrência de políticas públicas, notadamente os indicadores de processo, enquanto indicadores de resultado só se alteram após períodos de seguimento mais longo.

Com o uso destes parâmetros, foram identificados 110 municípios com este perfil de assistência. Como se observa também nos outros estados, a maior parte das cidades consistia de pequenos municípios, salvo poucas exceçōes. $\mathrm{Na}$ amostra, mais de $80 \%$ dos municípios possuíam menos de 10.000 habitantes ${ }^{16}$. A localizaçáo no Estado, bem como os perfis socioeconômicos, variou muito entre eles.

Os dados referentes ao Estado foram obtidos pela fonte de dados do Departamento de Informática do SUS ${ }^{4}$. Para avaliá-los na amostra, utilizaram-se, para a construçáo dos indicadores, dados do Sistema de Informaçóes Hospitalares (SIH), Sistema de Informaçóes sobre Mortalidade (SIM),
Sistema de Informaçóes Alimentares e Nutricionais (SISVAN), bem como informaçóes que alimentam o Sistema de Informaçōes em Atenção Básica (SIAB). Para se definir a população estudada, utilizaram-se dados do Sistema de Informações de Nascidos Vivos (SINASC), do CENSO, bem como as estimativas de bases populacionais utilizadas pelo DATASUS. O modo de construção dos indicadores é apresentado no Pacto pela Atençáo Básica ${ }^{14}$ e no Pacto pela Saúde de $2009^{15}$ e encontra-se disponível no DATASUS ${ }^{17}$, especificado nas notas técnicas.

Inúmeros indicadores do pacto foram levantados e condensados, mas, com finalidade de sintetizar, foram escolhidos os seguintes parâmetros para a pesquisa: coeficiente de mortalidade infantil, taxa de internação hospitalar por acidente vascular cerebral, taxa de internação por doença diarreica aguda em menores de 5 anos, percentagem de crianças com menos de 5 anos com baixo peso, percentual de nascidos vivos com 7 ou mais consultas de pré-natal, razão de exames citopatológicos em mulheres de 25 a 59 anos, percentual de cura de casos novos de tuberculose e percentual de cura de casos novos de hanseníase.

Ainda avaliando a efetividade da Estratégia Saúde da Família sobre outros ângulos, utilizou-se o percentual de famílias beneficiárias do Programa Bolsa Família acompanhadas pela atenção primária. $\mathrm{O}$ instrumento mostrou-se interessante na avaliação de saúde, uma vez que o indicador mede o acompanhamento efetivo de famílias que apresentam uma condição socioeconômica pior que a maior parte da população. Inclui-se, entre as avaliaçóes propostas pelo Programa, o seguimento do pré-natal quando existem gestantes nas famílias, o calendário vacinal e a avaliação nutricional das crianças com menos de 7 anos de idade.

Outros indicadores levantados, como o percentual de cobertura vacinal tetravalente, foram suprimidos do estudo por repetirem os achados que serão apresentados nos resultados.

A forma de cálculo de alguns indicadores, como a taxa de internaçôes hospitalares por diabetes mellitus e complicaçôes, sofreu alteraçôes por determinação do Ministério da Saúde muitas vezes ao longo dos anos. Portanto, a série histórica apresenta-se limitada por estes e outros fatores. Da mesma forma, com o intuito de se uniformizar os achados e minimizar possíveis vieses, alguns valores colocados para o Estado de São Paulo foram recalculados com parâmetros idênticos aos da amostra. Na taxa de internação por doença diarreica aguda, utilizaram-se dados disponíveis no DATASUS de internaçôes por "shiguelose", "amebíase", "gastroenterite e diarreia de origem infecciosa presumível" e "outras diarreias infecciosas", achados estes que se aproximaram muito dos valores consolidados no site do DATASUS. Não fosse feito 
tal ajuste para ambas as populaçôes (amostra e estado), os resultados estariam comprometidos.

$\mathrm{Na}$ análise dos dados, deve-se levar em conta que muitos municípios do Estado e da amostra não apresentavam dados referentes a determinado indicador em um ou mais anos. $\mathrm{O}$ problema se observa na avaliação do percentual de cura de pacientes com tuberculose bacilífera, bem como em outras situaçóes.

\section{Resultados}

A partir dos indicadores do Pacto pela Saúde, o primeiro indicador avaliado foi mortalidade infantil, pela sua importância em cenários nacionais e internacionais para se avaliar os perfis de saúde de uma população. Dados referentes a 2006 não se encontravam no portal do DATASUS, bem como os de 2010. Para a amostra, a média da mortalidade infantil para o ano de 2000 foi de 19,25 para mil nascidos vivos, período que apresentava variável cobertura da estratégia. No mesmo ano, a mortalidade no Estado foi de 17,33 para mil nascidos vivos, ou quase 2 pontos abaixo. Os valores, tanto do Estado quanto da amostra, apresentaram queda nos nove anos, sendo esta queda mais pronunciada para os municípios da amostra, como se vê pelas linhas de tendência na Figura 1. Para cada ano, no Estado, houve queda média de 0,48 na mortalidade infantil, enquanto que, na amostra, a queda anual foi de 0,63 , ou seja, $31,2 \%$ maior.

Como segundo indicador de resultado, utilizou-se a taxa de internação hospitalar por acidente vascular cerebral. A série histórica limitou-se aos últimos três anos pelo fato de que o Ministério da Saúde passou a utilizar, a partir deste período, a base populacional de 30 a 59 anos como populaçáo a ser estudada. As internações nos municípios da amostra foram maiores em relaçấo às do Estado em todos os anos (2008, 2009 e 2010) (Figura 2). No entanto, observou-se aumento nas internações no Estado de 0,47 internações por ano para 10.000 habitantes, o que não se viu na amostra.

Um terceiro indicador de resultado avaliado foi a porcentagem de crianças com menos de cinco anos com baixo peso, utilizando dados do SISVAN (Sistema de Vigilância Alimentar e Nutricional). Os dados da amostra e do Estado foram semelhantes para cada ano, não apresentando tendência de queda em ambas as populaçóes. A amostra apresentou proporção discretamente aumentada de crianças com baixo peso $(2,99 \%)$ em relação ao Estado $(2,87 \%)$ na média do período acompanhado, variação esta sem qualquer relevância em termos epidemiológicos.

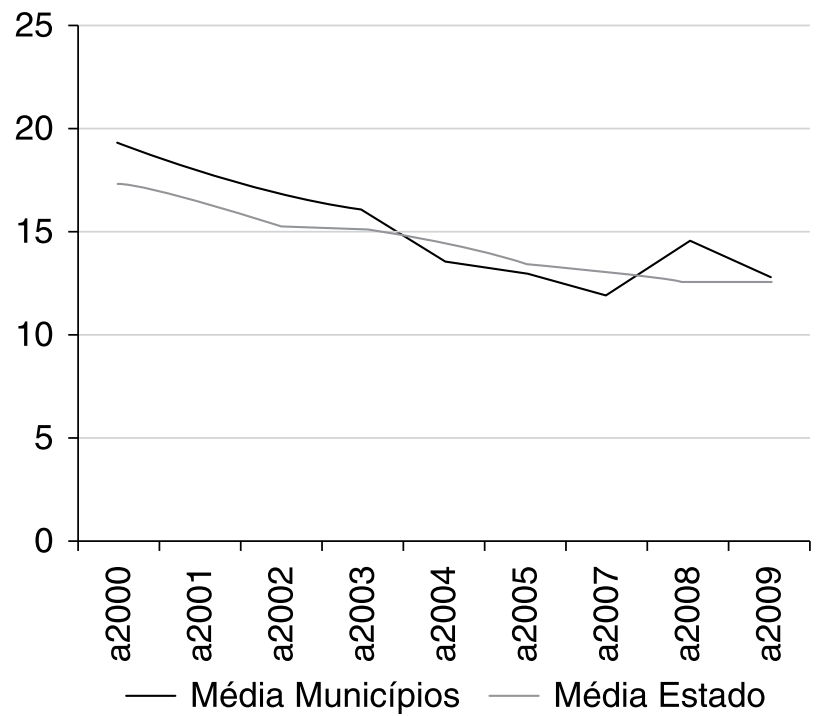

Figura 1. Coeficiente de Mortalidade Infantil no Estado de São Paulo. Fonte: DATASUS, SIM E SINASC, 2010.

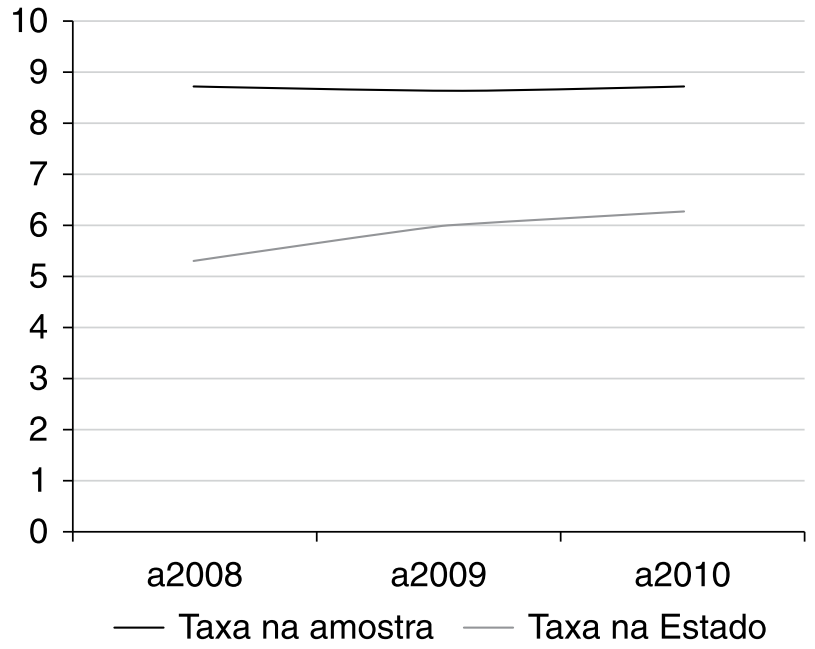

Figura 2. Taxa de internação por acidente vascular cerebral para cada 10.000 habitantes, na amostra e para o Estado de São Paulo, na população de 30 a 59 anos. Fonte: AlH e Estimativas Populacionais.

A taxa de internação de crianças com menos de 5 anos por doença diarreica aguda em cada ano estudado apresentou-se maior nos municípios da amostra, quando comparados aos resultados do Estado. Na média, a amostra obteve 9,09 internaçóes para cada 1.000 crianças, contra 4,34 internaçóes para cada 1.000 crianças no Estado. A tendência de queda nas internaçôes entre os municípios da amostra foi superior à encontrada no restante do Estado. Nos primeiros, observou-se queda de 0,29 internaçóes para 1000 crianças por ano, enquanto, nos últimos, a queda foi de 0,11 internaçôes por ano.

Como indicador de processo, também presente no Pacto pela Saúde, a porcentagem de nascidos vivos, cujas mães tiveram 7 ou mais consultas de pré-natal, apresentou-se 
um pouco maior entre os municípios da amostra, quando comparados à média estadual. A média encontrada foi de $72,6 \%$ dos nascidos com 7 ou mais consultas na amostra, contra 68,44\% entre os municípios do Estado. Em 2011, os valores foram, respectivamente, $80,21 \%$ contra $76,2 \%$. Em ambos os grupos, observa-se tendência, ao longo dos anos, de aumento de consultas de pré-natal entre os nascidos vivos.

A razão de exames citopatológicos para a população feminina de 25 a 59 anos, definida no "Pacto pela Saúde" como a razão dos exames citopatológicos nessas pacientes sobre a respectiva população alvo, mostrou-se maior entre os municípios da amostra em relaçáo às demais regiōes do Estado para cada ano estudado, estando a razão média em 0,29 exames para a população na amostra contra 0,15 no Estado, significando que, na amostra, a populaçáo alvo realiza quase duas vezes mais exames de rastreamento de câncer de colo do útero do que o restante do Estado. Por outro lado, observa-se na amostra uma queda da proporção de exames, o que se observa também no Estado, mas não na mesma proporção (Figura 3).

A proporçáo de cura de casos novos de tuberculose bacilífera apresentou-se maior em todos os anos da série histórica nos municípios da amostra, à exceção do primeiro ano. A média de cura na amostra para todos os anos foi de $76,17 \%$ dos casos, contra $62,46 \%$ do Estado, como bem se vê na Figura 4.

$\mathrm{Na}$ análise da proporção de cura de casos de hanseníase, observou-se uma diferença mais discreta, favorável aos indicadores da amostra $(90,86 \%$ vs $85,76 \%)$, para a média dos anos. Da mesma forma, nos municípios e no Estado, observa-se também uma queda na proporção de cura dos pacientes, alcançando os menores valores em 2010.

Em relaçáo ao percentual de famílias beneficiárias do Programa Bolsa Família acompanhadas pela atenção primária, ambos os grupos apresentam tendência de crescimento, porém no Estado observou-se um aumento discretamente maior do que na amostra. Por outro lado, em todos os anos, a amostra mostrou proporçáo de famílias de baixa renda acompanhadas quase $20 \%$ acima do restante do Estado (Figura 5).

\section{Discussão}

Para se atenuar possíveis vieses, foram utilizados os mesmos critérios de avaliação dos municípios da amostra e do restante do Estado de Sáo Paulo. Vale ponderar que os achados apresentam imperfeições decorrentes de falhas nas notificaçóes e registros feitos pelas equipes de saúde nos municípios, falhas estas que se verificam mais claramente quando se pesquisam

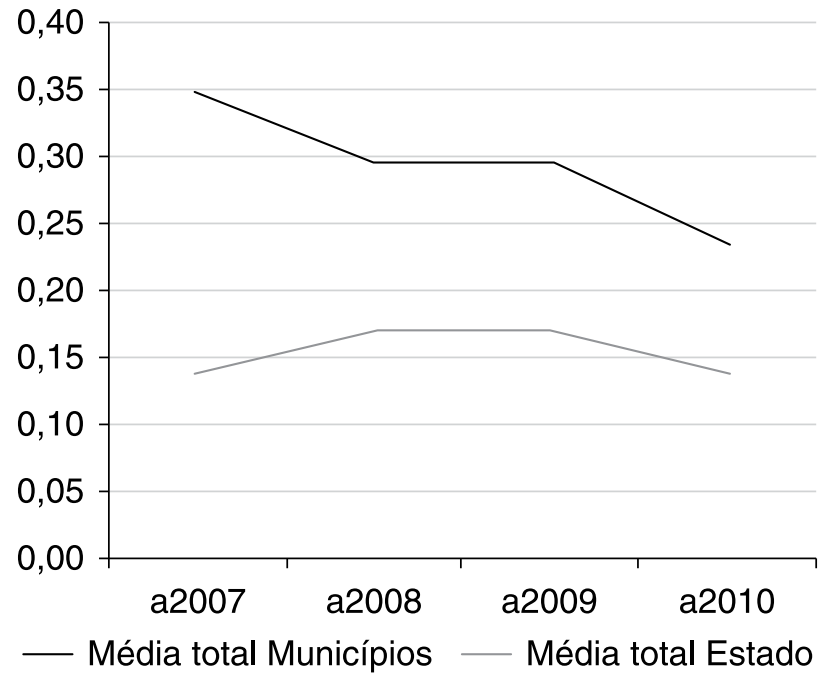

Figura 3. Razão de Exame Citopatológico para a População de 25 a 59 anos no Estado de São Paulo. Fonte: IBGE e SISCOLO.

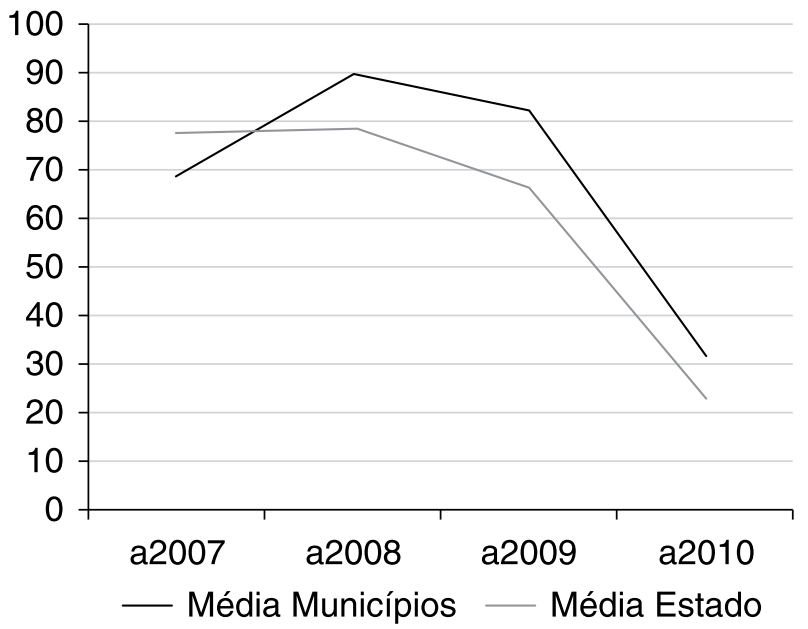

Figura 4. Percentual de Cura de Casos Novos de Tuberculose Bacilífera para o Estado de São Paulo. Fonte: SINAN.

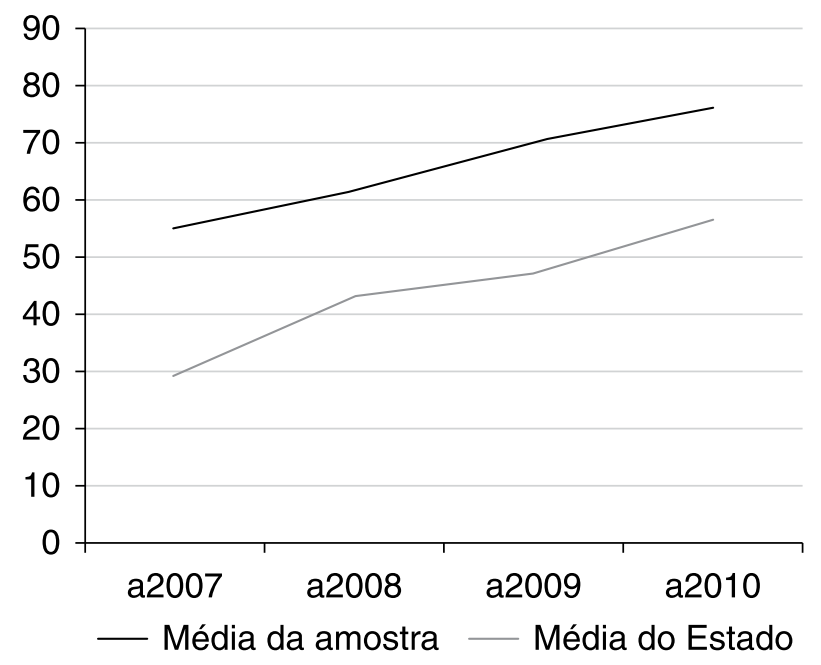

Figura 5. Percentual de Famílias com perfil saúde beneficiárias do Programa Bolsa Família acompanhadas pela APS. Fonte: SISVAN. 
os dados pelo DATASUS. Em decorrência de problemas nas esferas regionais, muitos municípios apresentam indicadores claros, enquanto outros náo divulgam resultados de alguns anos. Da mesma forma, pela mudança da prioridade de certos indicadores pelo Ministério da Saúde, o Departamento de Atenção Básica não divulga os dados de alguns anos. Ainda considerando as imperfeiçôes do estudo, pelo tamanho e heterogeneidade da amostra, os fatores de confusão que são particulares de cada cidade não puderam ser avaliados.

$\mathrm{O}$ estudo demonstrou que os municípios com maior cobertura da Estratégia Saúde da Família apresentaram tendência mais evidente de melhora no coeficiente de mortalidade infantil.

As internações em crianças com menos de 5 anos por doença diarreica foram mais frequentes nos municípios da amostra, mas apresentaram queda mensurável maior ao longo da série histórica. Nas internaçôes por acidente vascular cerebral, embora os municípios da amostra apresentem uma taxa maior, esta permaneceu estável durante a série histórica, enquanto o restante do Estado apresentou tendência ascendente. A proporção de crianças com baixo peso não apresentou diferença entre os municípios da amostra e o restante do Estado.

A interpretação de indicadores como as taxas de internação por acidente vascular cerebral e doença diarreica aguda, no entanto, deve ser cuidadosa, pois, onde há melhor acesso e assistência de mais qualidade, amplia-se a identificação dos agravos antes ocultos.

$\mathrm{Da}$ análise de todos os indicadores de resultado avaliados, se depreendeu a necessidade de se utilizar uma série histórica mais extensa.

Os indicadores de processo, que notadamente apresentam sensibilidade maior a alteraçóes nos padrôes de assistência, mostraram resultados mais convincentes para a amostra, sinalizando desempenho melhor da estratégia em vários processos priorizados pelo Ministério da Saúde. Entre eles, merece destaque a razáo de exames citopatológicos para a população de 25 a 59 anos, apresentando valores muito superiores às demais regiôes do Estado, e a proporção de cura de casos novos de tuberculose, que se mantém marcadamente melhor na amostra para todo o período estudado. A queda da razão de exames citopatológicos observada na amostra sinaliza, por outro lado, uma deterioração da atenção voltada especificamente para essa importante ação das equipes, perda de qualidade, que advém muitas vezes de uma menor cobrança dos gestores, em todas as suas instâncias.

Os resultados sinalizam a contribuição da ESF na melhora dos indicadores de saúde selecionados, apontando para a necessidade de maior seguimento para que sejam avaliados os indicadores de resultados. Impressões mais claras poderão ser tomadas após um período maior de estudo, considerando-se a lenta evolução da estratégia no Estado. A Estratégia Saúde da Família é modelo em constante construção e cabe aos partícipes a elaboração de estratégias mais efetivas. Tem-se também de forma clara que, apesar da ampla cobertura nas cidades da amostra e em muitos outros lugares do País, a estratégia não se desenvolve muitas vezes nas melhores condições por razóes diversas (cumprimento irregular dos horários pelos médicos, verbas escassas para a saúde...) e cabe aos gestores de esferas municipais, estaduais e federais o acompanhamento dos parâmetros estabelecidos.

\section{Referências}

1. Brasil. Ministério da Saúde. Secretaria Executiva. Programa Agentes Comunitários da Saúde - PACS. Brasília: Ministério da Saúde; 2001. [acesso em 10 maio 2011]. Disponível em http://bvsms.saude.gov.br/ bvs/publicacoes/pacs01.pdf

2. Lima PVPS, Khan AS, Silva LMR, Mayorga RD. O Programa de Agentes Comunitários de Saúde e os Indicadores de Saúde da Família do Estado do Ceará [on line]. IPECE; 2008. [acesso em 11 nov. 2011]. Disponível em: http://www2.ipece.ce.gov.br/encontro/ artigos_2008/26.pdf

3. Brasil. Conselho Nacional de Secretários de Saúde. SUS 20 anos Brasília: CONASS, 2009.[citado 25 nov 2011] Disponível em: http:// www.conass.org.br/arquivos/file/sus20anosfinal.pdf

4. Brasil. Ministério da Saúde. Departamento de Informática do SUS - DATASUS. Pacto pela Saúde - 2010/2011 - Brasil. versão 3.6 do Tabwin. Brasília: Ministério da Saúde; 2011 [acesso em 11 nov. 2011]. Disponível em: http://tabnet.datasus.gov.br/cgi/tabnet. exe?pacto/2010/cnv/pactbr.def

5. Brasil. Ministério da Saúde. Secretaria de Assistência à Saúde. Departamento de Atenção Básica. Aprimorando a Política Nacional de Atenção Básica. [acesso em10 maio 2011]. Disponível em: http:// portal.saude.gov.br/portal/arquivos/pdf/2d_060511.pdf

6. Starfield B, Shi L, Macinko J. Contribution of primary care to health systems and health. Milbank Q. 2005; 83(3): 457-502. http://dx.doi. org/10.1111/j.1468-0009.2005.00409.x

7. Macinko J, Guanais FC, Souza MFM. Evaluation of the impact of the Family Health Program on infant mortality in Brazil, 1990-2002. J Epidemiol Community Health. 2006; 60(1): 13-9. http://dx.doi. org/10.1136/jech.2005.038323

8. Macinko J, Souza MFM, Guanais FC, Simões CCS. Going to scale with community based primary care: an analysis of the family health program and infant mortality in Brazil, 1999-2004. Soc Sci Méd. 2007; 65: 2070-80.

9. Aquino R, Oliveira NF, Barreto ML. Impact of the family health program on infant mortality in Brazilian municipalities. Am J Public Health 2009; 99(1): 87-93. http://dx.doi.org/10.2105/ AJPH.2007.127480

10. Macinko J, Oliveira VB, Turci MA, Guanais FC, Bonolo PF, Lima-Costa MF. The influence of primary care and hospital supply on ambulatory care-sensitive hospitalizations among adults in Brazil, 1999-2007. Am J Public Health. 2011;101:1963-70. http://dx.doi.org/10.2105/ AJPH.2010.198887 
11. Mendonça CS, Harzheim E, Duncan BB, Nunes LN, Leyh W. Trends in hospitalizations for primary care sensitive conditions following the implementation of Family Health Teams in Belo Horizonte, Brazil. Health Policy Plan. 2012; 27(4): 348-355. http://dx.doi.org/10.1093/ heapol/czr043

12. Viacava F. Acesso e uso de serviços de saúde pelos brasileiros. Radis - Comunicação em Saúde. 2010; 96: 12-9 [acesso em 2 dez. 2011]. Disponível em: http://www4.ensp.fiocruz.br/radis/96/ capa.html

13. Roncalli AG, Lima KC. Impacto do Programa Saúde da Família sobre indicadores de saúde da criança em municípios de grande porte da região Nordeste do Brasil. Cienc Saúde Coletiva. 2006; 11(3): 713-24. http://dx.doi.org/10.1590/S1413-81232006000300018

14. Brasil. Ministério da Saúde. Portaria n 493, de 13 de março de 2006. Aprova a relação de indicadores da Atenção Básica - 2006, cujos indicadores deverão ser pactuados entre municípios, estados e Ministério da Saúde. Diário Oficial da República Federativa do Brasil, Brasília,13 mar. 2006. Seção 1, p. 45. [acesso em 11 nov. 2011].
Disponível em: http://www.saude.mg.gov.br/atos_normativos/ legislacao-sanitaria/estabelecimentos-de-saude/atencao-basica/ portaria_493.pdf

15. Brasil. Ministério da Saúde. Portaria GM nº 2.669, de 3 de novembro de 2009. Estabelece as prioridades, objetivos, metas e indicadores de monitoramento e avaliação do Pacto pela Saúde, nos componentes pela Vida e de Gestão, e as orientações, prazos e diretrizes do seu processo de pactuação para o biênio 2010 - 2011. Diário Oficial da República Federativa do Brasil, Brasília, 6 nov. 2009. Seção 1, p. 58-60.

16. Instituto Brasileiro de Geografia e Estatística - IBGE. Sinopse do Censo Demográfico 2010. Disponível em: http://www.censo2010.ibge.gov. $\mathrm{br} /$ sinopse/index. php?uf=35\&dados $=0$

17. Brasil. Ministério da Saúde. Departamento de Informática do SUS - DATASUS. Pacto pela Saúde - 2010/2011 - Brasil. Dados preliminares - Notas Técnicas. versão 3.6 do Tabwin. Brasília: Ministério da Saúde; 2011 [acesso em 11 nov 2011]. Disponível em: http://tabnet. datasus.gov.br/cgi/pacto/2010/pactdescr.htm 